M. Minnaert : Different upper air etfects may be located at different heights, Crochets, for instance, in one layer and other effect in a different layer.

M. A. ElLISON: There is certainly evidence of two or three layers, because the relaxation times come out differently for different effects, $2.5 \mathrm{~min}$. time-lag for crochets and 7 min. for S.E.A.

T. R. KAISER: The most important far-ultra-violet atmospheric 'window' which extends into the $\mathrm{D}$ region is likely to be the narrow one which coincides with the $\mathrm{L} \alpha$ radiation. However, I would point out that there are a multiplicity of windows in the wave-length range $900-1200 \AA$ which extend to heights below $80 \mathrm{~km}$. Until more is known of the solar spectrum in this region, one should not dismiss the possibility of some contribution to $\mathrm{D}$-region effects from ultra-violet radiation other than $\mathrm{L} \alpha$.

M. MinnaERT: Rocket observations during a flare would be very desirable for answering this and other questions.

\title{
9. ASSOCIATED COSMIC-RAY EFFECTS
}

\section{By H. Alfvén}

Solar flares are often associated with changes in cosmic-ray intensity, as first shown by Lange and Forbush. The change is always an increase and is distinctly different from the magnetic storm cosmic-ray effect which usually is a decrease in intensity.

The flare effect is most pronounced in the softest part of the cosmic-ray spectrum which is measured by the neutron counter technique developed by Simpson and others (the neutron counters are sensitive to primaries down to a few $\mathrm{GeV}$. whereas ionization chambers and G.M.-counters have very low sensitivity to primaries below 5 or ro GeV.). A small flare is associated with an increase of $\mathrm{I}$ or $2 \%$, but increases by several hundred percent have been measured in connexion with big flares.

Also with ionization chambers and G.M.-counters flare effects have been observed. Only four cases are known with increases of the order $10 \%$.

Cosmic-ray effects are largest at stations situated in regions where the Earth is reached by cosmic rays coming from the direction of the Sun and moving in Störmer orbits, as shown by Firor. However, there are several cases where cosmic-ray effects have been observed far outside these regions.

The cosmic-ray effect occurs almost simultaneously with the solar flare. The time lag is often less than one hour.

It.has been claimed that an upper limit to the solar magnetic field can be obtained from the flare effect. This is true only under the assumption that the solar magnetic field is a dipole field. If, on the other hand, the solar magnetic field (or rather the interplanetary field) has the more complicated form indicated by other electromagnetic phenomena and by the fine structure of the corona, the flare effect is understandable and not in conflict with a field with a polar strength of the order ro gauss. Also, the fact that the flare effects are observed outside Firor's regions indicates the existence of an interplanetary field strong enough to scatter the particles considerably.

There are two possible interpretations of the solar flare cosmic-ray effect. Either the flare effect is the release of cosmic-ray particles trapped in magnetic fields near the Sun, or it represents an actual generation of cosmic rays. In both cases the effect is probably caused by the magneto-hydrodynamic shockwaves produced by the flare proceeding upwards through the corona.

\section{DISCUSSION}

C. B. A. MCCUSKer: It is perhaps worth pointing out that Neher and Stern found a much weaker intensity of low energy $(<800 \mathrm{MeV}$.) particles in I954, a year of low solar activity, than in $\mathbf{1 9 5 1 .}$ 
M. N. SaHa: Primary cosmic rays consist of proton and helium particles mainly, but neon particles are also found to occur quite strongly. Now the solar spectrum does not show the slightest trace of any lines of neon, though these occur in a very favourable region (red). So we have to admit that neon does not occur in the sun. How are we then to account for the occurrence of neon-nuclei in the primary cosmic-ray spectra?

H. ALFVÉn: The origin of cosmic-ray radiation is a complicated phenomenon, and a full discussion would take us too far now. The direct connexion between flares and increases in cosmic radiation indicates that at least part of the cosmic radiation is accelerated near the Sun. But it is an open question whether all the cosmic radiation originates near the Sun.

R. Q. Twiss: Will Prof. Alfvén comment on the time delay between the occurrence of the flare and the increase in cosmic-ray intensity at the Earth?

H. Alfvén: If the excess cosmic-ray flux is produced at the same time as the optical flare, one must attribute the delay to irregular magnetic fields between the Sun and the Earth which increase the effective path for charged particles with a factor $\sim 3-10$. Alternatively, if the excess is produced by a magnetic acceleration process following the flare, the delay could be attributed to the finite time needed for the magnetic energy to build up.

M. Minnaert : I believe that balloon observations during flares have indicated increases of cosmic radiation of the order of $200 \%$.

H. Alfvén: Yes. Upon the whole, the increases are more pronounced for smaller energies, such as registered by neutron counter and in balloon measurements.

M. Minnaert: Balloon measurements during flares are clearly important.

A. SCHLÜTER: Generation of cosmic radiation on the Sun seems to be proved only in the case of four big increases in cosmic radiation. For all other cases ('small flare effects') the interpretation of the increases as modulation of the existing cosmic radiation is not excluded. A continuous transition from 'small' to large flare effects has not been established as yet. Assuming modulation, the contribution of the Sun to the total cosmic-ray flux would be very small.

H. ALFVÉN: Yes, there is a possibility that flares only release cosmic rays available in reservoirs near the Sun.

D. H. Menzel: With reference to Dr Saha's remark, I should say that the solar abundance of neon is very uncertain because of the unfavourable location of its fundamental lines.

K. O. KiepenheUeR: It is dangerous to draw too far-reaching conclusions from connexions between flare observations and cosmic radiation. There are many complicated processes taking place in the vicinity of flares.

\section{ro. HYPOTHESES ON THE ORIGIN OF FLARES}

By A. UNSÖLD

\section{Some Observational Characteristics}

A theory of flares should explain the following observations:

(a) The rise to peak intensity within $\sim 5 \mathrm{~min}$.

(b) The time of decay being independent of the time of rise; mostly $\sim \mathrm{r} \mathrm{hr}$.

(c) Bright flares occur mostly in spot groups of maximum development; they are frequently connected with bright flocculi or plages faculaires.

(d) Flares seem to vary between $\sim$ I sec. diameter and $\sim 5^{-I 5} \mathrm{~min}$. duration and 5000 millionth of a hemisphere and several hours duration.

(e) Flares may occur in quite different levels; Ellerman's 'bombs' are probably below the photosphere, limb flares in the prominence level. 